\title{
New alternatively spliced variant of prostate-specific membrane antigen PSM-E suppresses the proliferation, migration and invasiveness of prostate cancer cells
}

\author{
KAI-YUAN CAO ${ }^{1-4, *}$, LIN XU $^{2-4, *}$, DING-MEI ZHANG ${ }^{2,4}$, XIAO-MING ZHANG ${ }^{2}$, TIAN ZHANG ${ }^{2}$,

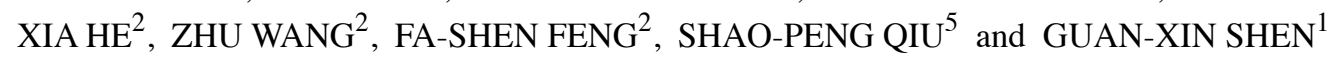 \\ ${ }^{1}$ Department of Immunology, Tongji Medical College, Huazhong University of Science and Technology, \\ Wuhan 430030; ${ }^{2}$ Research Center for Clinical Laboratory Standard, Zhongshan School of Medicine, ${ }^{3}$ Department of \\ Microbiology, Zhongshan School of Medicine, ${ }^{4}$ Key Laboratory of Tropical Disease Control, Ministry of Education, \\ ${ }^{5}$ Department of Urology, The First Affiliated Hospital, Sun Yat-Sen University, Guangzhou 510080, P.R. China
}

Received October 18, 2011; Accepted December 27, 2011

DOI: $10.3892 /$ ijo.2012.1358

\begin{abstract}
PSM-E is a newly discovered alternatively spliced variant of prostate-specific membrane antigen (PSMA). In the current study, its role on the proliferation, invasiveness and migration in prostate cancer cell lines was analyzed. PSM-E and PSMA (as a comparison) eukaryotic expression vectors pcDNA3.0/PSM-E and pcDNA3.0/PSMA were constructed, validated by RT-PCR and Western blotting, and PSMA/PSM-E overexpression PC-3 cell models were built. Gene interference was used to block PSMA and the expression of its splice variants in LNCaP cells. Three shRNA fragments were synthesized against PSMA, cloned into the vector pSilencer 2.1-U6-neo, their interference effect was evaluated by RT-PCR and Western blotting, and pSilencer 2.1-U6-neo-shRNA3 (named p-shRNA3) was chosen in further analyses. Growth curves were drawn to observe the proliferation change, which showed that PSM-E had the potential to suppress proliferation $(\mathrm{P}<0.05)$, but no significant change was observed in PSMA/PC-3 cells and in PSMA/PSM-E interfering LNCaP cells ( $\mathrm{P}>0.05)$. Cross-river test showed that the migration speeds of PSM-E/PC-3 and PSMA/PC-3 were both significantly slower than the vector negative control, and faster in p-shRNA3 interfering LNCaP
\end{abstract}

Correspondence to: Professor Guan-Xin Shen, Department of Immunology, Tongji Medical College, Huazhong University of Science and Technology, Wuhan 430030, P.R. China

E-mail: guanxin_shen@yahoo.com.cn

Professor Kai-Yuan Cao, Research Center for Clinical Laboratory Standard, Zhongshan School of Medicine, Sun Yat-sen University, Guangzhou 510080, P.R. China

E-mail: caoky@mail.sysu.edu.cn

* Contributed equally

Key words: prostate-specific membrane antigen, splice variant, PSM-E, prostate cancer, RNA interference cells compared with its vector negative control $(\mathrm{P}<0.05)$, and no significant difference existed between PSM-E/PC-3 and PSMA/PC-3 ( $\mathrm{P}>0.05)$. Transwell assay showed that the invasive cells of both PSMA/PC-3 and PSM-E/PC-3 were fewer compared to the vector negative control $(\mathrm{P}<0.05)$, and the invasive suppression effect of PSM-E was weaker than PSMA $(\mathrm{P}<0.05)$, and accordingly, invasiveness of interfering LNCaP cells was enhanced compared with the vector negative control $(\mathrm{P}<0.05)$. These results showed that PSM-E could suppress proliferation, migration and invasiveness of prostate cancer cells. Its suppression effect on cell proliferation is stronger compared to PSMA and the suppression effect on invasiveness is weaker than that of PSMA.

\section{Introduction}

Prostate cancer $(\mathrm{PCa})$ is one of the most common malignant tumors and is the second leading cause of mortality from cancer among males in Europe and America (1). Its incidence has increased worldwide in recent years. Because the clinical symptoms of PCa are not always obvious, many patients were found to have metastasis by the time of diagnosis (2). Therefore, it is very important to explore the biological behavior of its developing process.

Prostate-specific membrane antigen (PSMA) is a type II transmembrane glycoprotein whose gene maps to chromosome $11 \mathrm{p} 11-11 \mathrm{p} 12$, and is expressed on the surface of both normal and PCa cells (3-8). It consists of 750 amino acids and has a molecular weight of $100 \mathrm{kDa}$ after glycosylation. It has two important enzymatic functions, namely, folate hydrolase and NAALADase (3-7). Its expression in prostate cells is up to 50 -fold higher than in other expressing tissues, such as kidney, bladder, brain, liver, and intestine $(9,10)$. Importantly, PSMA is overexpressed in most PCa tissues, and also highly expressed in the neovasculature of a variety of solid tumors (11-13), indicating that PSMA may correlate to cancer progression. Due to its high tissue specificity and pathological specificity, PSMA has been a potential target for diagnosis and treatment of $\mathrm{PCa}$ $(14,15)$. 
Alternative splicing is a common phenomenon that can be observed in both benign and malignant tissues $(16,17)$. A few PSMA splice variants have been found, including PSM', PSM-C, PSM-D, and PSM-E (18,19). PSM-E is an alternatively spliced variant we found previously that is different from other known variants (GenBank locus EF488811.2) (19). Its cDNA includes 2653 nucleotides, has a 97 bp new exon inserted into the site between 379-380 of PSMA cDNA, and a 93 bp deletion located at the region of 2232-2324 nucleotides of PSMA cDNA (19). Bioinformatics analysis showed that the PSM-E cDNA encodes a glycoprotein of 704 amino acids, with a possible signal peptide of 1-21aa, 150-250aa as a protease associated domain (PA domain), 355-549aa as a metallopeptidase domain, and 597-703aa as a TFR dimer domain. Though structurally similar, it may be functionally different from PSMA. We have found that PSM-E is specifically overexpressed in PCa, and has even higher tissue specificity and pathological specificity than PSMA (19), but its biological function and its possible effect in PCa progression is still unknown. Our previous studies suggest it may be a candidate for a potent molecular marker for the measurement of PCa progression. So, in this study, using migration and invasiveness tests of PSM-E overexpression and interfering PCa cell models, we explored the effect of PSM-E and PSMA on the proliferation, invasiveness and migration of PCa cell lines in vitro. Our findings may contribute to revealing the potential role of PSM-E and PSMA in cancer progression, and may have some significance in understanding the biological role of PSM-E and PSMA.

\section{Materials and methods}

Cell lines. PCa cell lines LNCaP and PC-3 were obtained from American Type Culture Collection, and cultured in RPMI-1640 (Gibco-BRL, Invitrogen Co., Grand Island, NY, USA), 1 mM sodium pyruvate, $100 \mu / \mathrm{ml}$ penicillin $\mathrm{G}, 100 \mu \mathrm{g} / \mathrm{ml}$ streptomycin (Sigma, Ronkonkoma, NY, USA), $10 \%$ fetal bovine serum (FBS, Tianjin Hao Yang Biological Manufacture Co. Ltd, Beijing, China). Recombinant plasmid pMD18-T/PSM-E and pMD18-T/PSMA were constructed previously in our laboratory. shRNA expressing vector pSilencer 2.1-U6-neo was a product of Ambion Inc., Austin, USA.

Construction of PSMA/PSM-E eukaryotic expression vectors. The PSM-E and PSMA cDNA was obtained, respectively, from recombinant plasmid pMD18-T/PSM-E and pMD18-T/ PSMA by common primers (19). The forward primer was ATA GGATCC GAG ATG TGG AAT CTC CTT CAC, which contained the ribosome entry site and BamHI restriction enzyme site; and the corresponding reverse primer was ATA CTCGAG TCC TCT TAG GCT ACT TCA CTC, corresponding to the 21-nucleotide near the $3^{\prime}$ end and containing XhoI restriction site. PCR amplification was carried out using high fidelity Platinum Taq DNA polymerase (Takara Biotechnology Co., Ltd, Dalian, China) in $50 \mu 1$ total volume. PCR procedure was as follows: $94^{\circ} \mathrm{C}$ for 5 min, cycle $1 ; 94^{\circ} \mathrm{C}$ for $30 \mathrm{sec}, 54.2^{\circ} \mathrm{C}$ for $30 \mathrm{sec}, 72^{\circ} \mathrm{C}$ for $150 \mathrm{sec}$, cycle 2 through 35; then extended for $10 \mathrm{~min}$ at $72^{\circ} \mathrm{C}$. The expected fragment length of PSMA and PSM-E was 2279 and 2283, respectively. The products were recovered, restricted by BamHI and XhoI (Takara), ligated into pcDNA3.0 vector and transformed into
E. coli DH5 $\alpha$. Recombinant plasmids were confirmed by BamHI and XhoI restriction as well as DNA sequencing.

Construction of pSilencer 2.1-U6-neo-shRNA expressing vectors. The siRNA was designed by online design software siRNA Target Finder of Ambion. (http://www.ambion. com/techlib/mist/siRNAfinder.htmL). 3 shRNA fragments were obtained, $19 \mathrm{nt}$ of length, GC concentration as $38.1 \%$, $52.4 \%, 47.6 \%$, respectively, with the upstream restriction site as BamHI, and downstream restriction site as HindIII. The complementary sites were on 869 bp, 1589 bp and 2194 bp of PSMA cDNA sequence, respectively. The loop sequence was TTCAAGAGA, and the shRNA was designed as BamHI+sense +loop+antisense+termination signal+HindIII, and synthesized by Invitrogen, Shanghai, China. Synthesized complementary DNA oligonucleotide chains were dissolved by annealing buffer and diluted into $1 \mu \mathrm{g} / \mu \mathrm{l}$, respectively. The sense and antisense oligonucleotide chains were annealed together to get dsDNA, then the dsDNA were ligated into linearized pSilencer2.1-U6-neo vector by T4 DNA ligase (Takara), the recombinant vectors then transformed into $E$. coli DH5 $\alpha$, and the positive clones were selected by ampicillin (Sigma) and identified by BamHI and HindIII restriction and DNA sequencing (Takara). pSilencer 2.1-U6-neo-NC, the negative control, was supplied by Ambion.

The expression of PSMA and PSM-E protein in PC-3 cells. PC-3 cells were tranfected by identified recombinant pcDNA3.0/PSMA and pcDNA3.0/PSM-E, respectively, with Lipofectamine $^{\mathrm{TM}} 2000$ (Invitrogen Co., CA, USA). Vector negative control was PC-3 cells transfected by pcDNA3.0 plasmid. The positive recombinant cell clones were selected by G418 (Sigma), and the optimal concentration $(500 \mu \mathrm{g} / \mathrm{ml})$ was decided by the dose-response curve drawn previously. G418 resistant clones were expanded, collected, and the expression of target proteins was identified by RT-PCR, immunofluorescence and Western blotting. The PSMA specific forward primer used in RT-PCR identification was: 5'-GGA GGG ATG GTG TTT GAG C-3', and the corresponding reverse primer was: 5'-CTG CCT GGT AAC CCT AAT GG-3'. The expected product length of PSMA was $312 \mathrm{bp}$. The forward primer for PSM-E identification was: 5'-GCC AAC TGC AAG GTC TAA TG-3', and the reverse primer: 5'-CCT GCT AAA TGT GGT ATC TGT G-3'. The expected product of PSM-E was $225 \mathrm{bp}$. PCR procedure was: $94^{\circ} \mathrm{C}$ for $3 \mathrm{~min}$, cycle $1 ; 94^{\circ} \mathrm{C}$ for $30 \mathrm{sec}, 55^{\circ} \mathrm{C}$ for $30 \mathrm{sec}, 72^{\circ} \mathrm{C}$ for $30 \mathrm{sec}$, cycle 2 through 30 ; then extended for $5 \mathrm{~min}$ at $72^{\circ} \mathrm{C}$. $\beta$-actin was also amplified as an internal control. The forward primer for $\beta$-actin was: 5'-GCA TGG AGT CCT GTG GCA TCC-3', and the reverse primer: 5'-CAT TTG CGG TGG ACG ATG GAC-3'. The product length of $\beta$-actin was $319 \mathrm{bp}$ and its PCR procedure was the same as that of PSMA and PSM-E. PCR products were identified by $1 \%$ agarose gel electrophoresis. The monoclonal antibody (YPSMA, ab19071; Abcam Inc., Cambridge, MA, USA) used in indirect immunofluorescence and Western blotting recognized 716-723 amino acid residues of the $\mathrm{C}$ terminus of PSMA. Cells were stained by FITC-labeled secondary goatanti-mouse antibody (Sigma) and counterstained by $0.02 \%$ Evan's blue. The antibody dilution ratio used in Western blotting was 1:1000 and in immunofluorescence 1:100. 
The pSilencer 2.1-U6-neo-shRNA inhibition of PSMA expression in LNCaP cells. LNCaP cells were seeded onto 24-well plates (Corning Incorporated Costar, Corning, NY, USA) at $2.0 \times 10^{5}$ cells/well and cultured for $24 \mathrm{~h}$. The recombinant pSilencer 2.1-U6-neo-shRNA vectors (3 recombinant vectors carrying different shRNA) and the negative control pSilencer 2.1-U6-neo-NC were trasfected into LNCaP cells using Lipofectamine $^{\mathrm{TM}} 2000$ (Invitrogen). The positive recombinant clones were selected by G418 (Sigma) for 14 days. The optimal concentration $(700 \mu \mathrm{g} / \mathrm{ml})$ was decided by the dose-response curve drawn previously. Stable G418 resistant clones of the four vectors (3 recombinant vectors and the negative control) were expanded, collected, and shRNA interference effect on PSMA and its splice variants was evaluated by RT-PCR and Western blotting. The recombinant vector sequencing primers were used first in RT-PCR to confirm transfection success. The forward primer was: 5'-GTT TTC CCA GTC ACG AC-3', and the reverse primer was: 5'-GAG TTA GCT CAC TCA TTA GGC-3'. Expected product length was 546 bp. Then, after transfection confirmation, PSMA and its splice variants were amplified, with the consensus forward primer: 5'-TAC CAC ATT TAG CAG GAA CAG AAC-3', and the consensus reverse primer: 5'-AAC CAT CTG GAT AGG ACT TCA CC-3'. The expected product length of PSMA series was 498 bp. $\beta$-actin served as an internal control (expected product length $318 \mathrm{bp}$ ). PCR procedure was: $94^{\circ} \mathrm{C}$ for $5 \mathrm{~min} ; 94^{\circ} \mathrm{C}$ for $40 \mathrm{sec}, 55^{\circ} \mathrm{C}$ for $40 \mathrm{sec}, 72^{\circ} \mathrm{C}$ for $45 \mathrm{sec}, 30$ cycles; then extended for $3 \mathrm{~min}$ at $72^{\circ} \mathrm{C}$. PCR products were identified by $1.5 \%$ agarose gel electrophoresis. The monoclonal antibody (YPSMA, ab19071) used in Western blotting was product of Abcam, dilution ratio 1:1000, and detected by chemiluminescence. The resulting gels from RT-PCR and Western blotting were scanned and calculated by the software Image Pro Plus. The mRNA and protein relative quantity were calculated by the following formula: mRNA (or protein) relative quantity = target band gray scale/ $\beta$-actin band gray scale. The inhibition rate calculation formula was: mRNA (or protein) inhibition rate = 1-(psiRNA mRNA relative quantity/pNC relative quantity).

Growth curve analysis. The identified stable transfected PC-3 and LNCaP cells (pcDNA3.0/PC-3, PSMA/PC-3, PSM-E/ PC-3 transfected PC-3 groups, 3 pSilencer 2.1-U6-neo-shRNA transfected LNCaP groups) and the negative control groups (non-transfected PC-3 cells and pSilencer 2.1-U6-neo-NC transfected LNCaP cells) were collected, digested by $0.25 \%$ trypsin and counted, then seeded onto 24-well plates (Corning Incorporated Costar) at the initial density of $2 \times 10^{4}$ cells/well. Transfected groups were continuously selected by $300 \mu \mathrm{g} / \mathrm{ml}$ G418. Cells were counted every 24 h for 5 days, each time counted 3 duplicate wells, using Typan blue staining to exclude dead cells. The growth curves were drawn for each group. Cell doubling time was calculated by the formula: $\mathrm{T}_{\mathrm{D}}=\operatorname{tx} \lg 2 / \lg \left(\mathrm{N}_{\mathrm{t}} /\right.$ $\mathrm{N}_{0}$ ).

Cross-river test. The pcDNA3.0/PC-3, PSMA/PC-3, PSM-E/ PC-3 and PC-3 negative control cell groups, and p-shRNA3 transfected, p-NC transfected and LNCaP negative control cell groups were inoculated into 6-well plates (Corning Incorporated Costar). When the bottom was fully covered by a monolayer of cells, a straight line was scratched through the center of the well using a $20 \mu \mathrm{l}$ tip. Flushing the stripped cells out of the line. The width of the scratched line was measured and the line was observed continuously until it was re-covered by adjacent cells. The time when the line was covered is reported as the migration time. For each group, the test was repeated 3 times to calculate the corresponding average scratching distance and migration time. The cell migration rate was calculated by: Migration velocity $(\mu \mathrm{m} / \mathrm{h})=$ scratching distance/migration time.

Transwell invasiveness test. The 24-well transwell chamber was a product of Corning Corporation. Fibronectin (10 $\mu \mathrm{l})$ was applied on the under layer of the chamber membrane, and on the upper layer of the membrane, after washing the wells with PBS 3 times, $50 \mu \mathrm{l}$ of Matrigel (dilution 1:50) was applied and allowed to solidify at $37^{\circ} \mathrm{C}$. BSA $(50 \mu \mathrm{l})$ (concentration $10 \mathrm{~g} / \mathrm{l}$ ) was applied for blockage of binding sites and incubated at $37^{\circ} \mathrm{C}$ for $30 \mathrm{~min}$. After incubation, the remaining liquid was removed, and $200 \mu \mathrm{l}$ of cell suspension (PC-3 cells and LNCaP cells) at the concentration of $1 \times 10^{5}$ cells $/ \mathrm{ml}$ was added to the upper layer of the transwell chamber. RPMI-1640 medium (600 $\mu \mathrm{l})$ supplemented with $1 \%$ FBS and $1 \%$ bovine serum albumin (BSA) was added to the underlayer of the chamber. The transwell chambers were incubated for $24 \mathrm{~h}$ at $37^{\circ} \mathrm{C}, 5 \% \mathrm{CO}_{2}$. After incubation, the culture medium was discarded, and the chamber was washed to remove cells that did not cross the membrane. The membrane was fixed in methanol for $5 \mathrm{~min}$, and then the cells crossing the membrane were stained by Wright-Giemsa staining solution for 8-10 min and counted. For each group, 3 duplicate wells were counted. The invasiveness index was calculated by: invasiveness index = (invasive cell number of experimental group/ invasive cell number of control group) x100\%.

Statistical analysis. SPSS11.5 was used for statistical analysis. Measurement data are shown as mean \pm SD. One-factor analysis of variance was used in data analysis between multiple groups. A value of $\mathrm{P}<0.05$ was considered statistically significant.

\section{Results}

Construction of PSMA and PSM-E overexpression and interfering cell models. PSMA-pcDNA3.0 and PSM-E-pcDNA3.0 eukaryotic expression vectors were successfully constructed and confirmed by restriction and sequence analysis, transfected PC-3 cells, named respectively as PSMA/PC-3 and PSM-E/PC-3, to construct PSMA and PSME overexpression PC-3 cell models. Their expression in transfected PC-3 cells was identified by RT-PCR and immunofluorescence (Figs. 1 and 2). Three shRNAs were obtained and synthesized, and the corresponding pSilencer 2.1-U6-neo-shRNA expressing vectors, named respectively as p-shRNA1, p-shRNA2 and p-shRNA3 were constructed, identified by enzyme restriction, DNA sequencing, and their transfection success was verified by RT-PCR (data not shown), and their expression in LNCaP cells was confirmed by RT-PCR and Western blotting (Fig. 3). The mRNA inhibition rate of p-shRNA1, 2, and 3 was $33.15 \%$, $9.26 \%$ and $41.97 \%$, respectively. The protein inhibition rate of p-shRNA1, 2, and 3 was $26.26 \%, 6.47 \%$ and $40.69 \%$, respectively. The mRNA and protein inhibition rate of the negative 


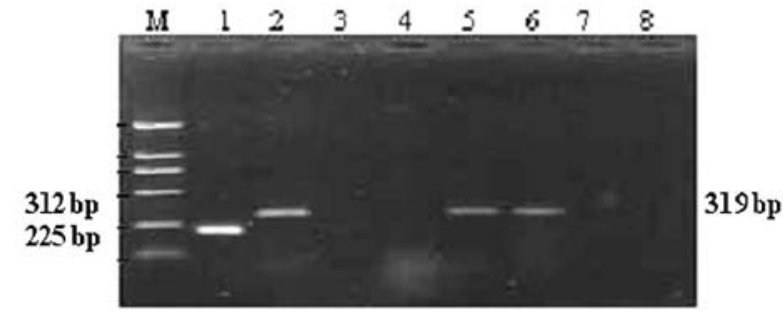

Figure 1. RT-PCR identification of PSMA and PSM-E expression in transfected PC-3 cells. The expression of PSMA, PSM-E was identified by RT-PCR using specific primers. $\beta$-actin was amplified as an internal control. The expected product length of PSMA was $312 \mathrm{bp}$, PSM-E was $225 \mathrm{bp}$, and $\beta$-actin was $319 \mathrm{bp}$. Lane M, DNA marker (2000, 1000, 750, 500, 250 and $100 \mathrm{bp}$ ); Lane 1, recombinant cell clone of PSM-E/ PC-3 (225 bp+); lane 2, recombinant cell clone of PSMA/PC-3 (312 bp+); lane 3, vector negative control of pcDNA3.0/PC-3 (225 bp-); lane 4, vector negative control of pcDNA3.0/PC-3 (312 bp-); lane 5/6, PCR internal control $\beta$-actin of pcDNA3.0/PC-3 (319 bp+) and lane 7/8, PCR negative controls.
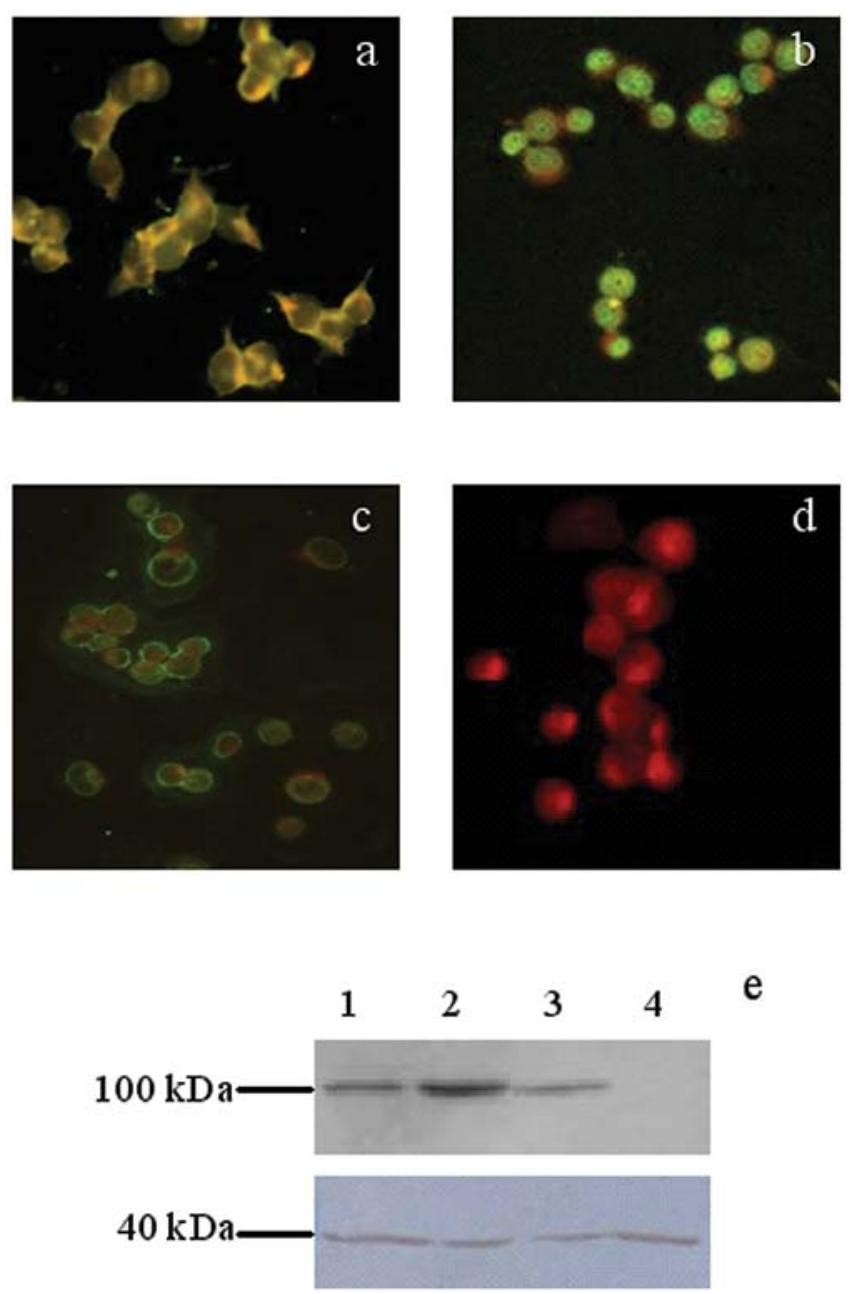

Figure 2. Indirect immunofluorescence and Western blotting identification of PSMA and PSM-E expression in transfected PC-3 cells. Cells were stained by PSMA specific monoclonal antibody and FITC-labeled secondary goatanti-mouse antibody (Sigma Co, USA) and counterstained by $0.02 \%$ Evan's blue. (a) Immunofluorescence of positive control of LNCaP cells (x200). (b) Immunofluorescence of PSMA/PC-3 (x200). (c) Immunofluorescence of PSM-E/PC-3 (x200). (d) Immunofluorescence of vector negative control pcDNA3.0/PC-3 (x200). (e) Western blotting identification of PSMA and PSM-E transfected PC-3 cells. Upper panel, PSMA/PSM-E; lower panel, $\beta$-actin. Lane 1, PSMA/PC-3 cells; lane 2, LNCaP cells; lane 3, PSM-E/PC-3 cells; lane 4, pcDNA3.0/PC-3 cells. a
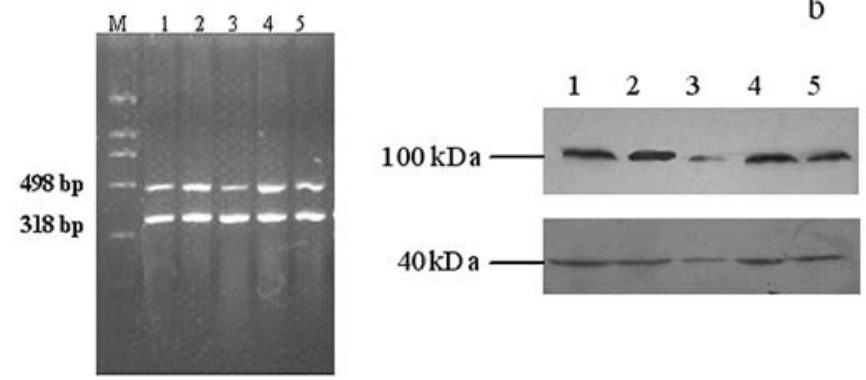

Figure 3. RT-PCR and Western blotting identification of p-shRNA interference effect on PSMA expression in LNCap cells. The cDNA of pSilencer 2.1-U6-neo-shRNA1,2,3 and pSilencer 2.1-U6-neo-NC transfected LNCap cell clones were amplified using universal primers of PSMA/PSMA splice variants to observe the mRNA interference effect. Western blotting was used to identify the protein interfering effect. $\beta$-actin served as an internal control. (a) RT-PCR identification of p-shRNA interference effect. Expected product length of $\beta$-actin was 318 bp and the PSMA series $498 \mathrm{bp}$. Lane M, DNA marker (2000, 1000, 750, 500, 250 and 100 bp). Lane 1, p-shRNA1; lane 2, p-shRNA2; lane 3, p-shRNA3; lane 4, negative control non-transfected LNCaP cells and lane 5, vector negative control p-NC. (b) Western blot analysis of PSMA and PSM-E expression. Upper panel, PSMA/PSM-E; lower panel, $\beta$-actin. Lane 1 , p-shRNA1; lane 2 , p-shRNA2; lane 3, p-shRNA3; lane 4, negative control LNCaP cells; lane 5, vector negative control p-NC.

control (p-NC group) was $10.1 \%$ and $7.97 \%$, respectively. Therefore, $\mathrm{p}$-shRNA1, and p-shRNA3 showed obvious inhibition effect at both mRNA and protein level $(\mathrm{P}<0.05$ compared with negative control). So, p-shRNA3 was chosen for the following proliferation, migration and invasiveness tests in LNCaP cells as it showed the maximum inhibition effect.

Proliferation analysis of PSM-E/ PSMA overexpressed PC-3 cells and interfering LNCAP cells. No significant change of proliferation was observed in PSMA/PC-3 overexpression cells and PSMA/PSM-E interfering LNCaP cells ( $\mathrm{P}>0.05$, Table I), but in PSM-E/PC-3 cells, cell proliferation was significantly suppressed $(\mathrm{P}<0.05$, Table I). There was no significant difference between PSMA/PC-3 cells and negative control/vector negative control cells ( $\mathrm{P}>0.05$, Fig. 4a), indicating that expression of PSMA did not obviously change PC-3 cell proliferation. However, the cell doubling time of PSM-E/PC-3 cells was significantly longer compared with vector negative control and PC-3 negative control groups $(\mathrm{P}<0.05$, Fig. $4 \mathrm{a})$, showing that PSM-E could suppress the proliferation of PC-3 PCa cells. Although compared with non-transfected LNCaP cells and p-NC group, no significant difference of proliferation was observed in p-shRNA3 group ( $\mathrm{P}=0.12$, Table I, Fig. 4b), it showed a trend of cell doubling time shortening. Therefore, PSM-E showed a certain effect of proliferation suppression of PCa cells, which may be covered by PSMA and other alternative spliced variants.

Migration analysis of PSM-E/PSMA overexpressed PC-3 cells and interfering LNCAP cells. The migration speeds of PSM-E/PC-3, PSMA/PC-3, pcDNA3.0/PC-3 vector negative control and PC-3 negative control groups were 21.3 \pm 2.08 , $21.0 \pm 3.61,39.0 \pm 2.65$ and $40.0 \pm 2.65 \mu \mathrm{m} / \mathrm{h}$, respectively (Table II). Similar to that of PSMA, PSM-E showed the effect of migration suppression, and the migration speed was significantly slower compared with the vector negative 
Table I. Cell doubling time of PSM-E/PSMA overexpressed and interfering prostate cancer cells.

\begin{tabular}{|c|c|c|c|c|c|c|c|}
\hline & PC-3 & pcDNA3.0/PC-3 & PSMA/PC-3 & PSM-E/PC-3 & $\mathrm{LNCaP}$ & $\mathrm{p}-\mathrm{NC}$ & p-shRNA3 \\
\hline Cell doubling time $(\mathrm{h})$ & $39.91 \pm 2.45$ & $40.75 \pm 3.93$ & $43.49 \pm 9.11$ & $49.14 \pm 3.34$ & $55.38 \pm 7.22$ & $58.64 \pm 4.43$ & $50.76 \pm 8.21$ \\
\hline P-value & 0.77 & & 0.66 & $0.04^{\mathrm{a}}$ & 0.64 & & 0.12 \\
\hline
\end{tabular}

The P-values of PC-3, PSMA/PC-3 and PSM-E/PC-3 groups were the P-value compared with the pcDNA3.0/PC-3 group (vector negative control), and $\mathrm{P}$-values of $\mathrm{LNCaP}$ and $\mathrm{p}$-shRNA3 group were the $\mathrm{P}$-value compared with the $\mathrm{p}$-NC group (vector negative control). ${ }^{\mathrm{P}} \mathrm{P}<0.05$ compared with vector negative control.

a

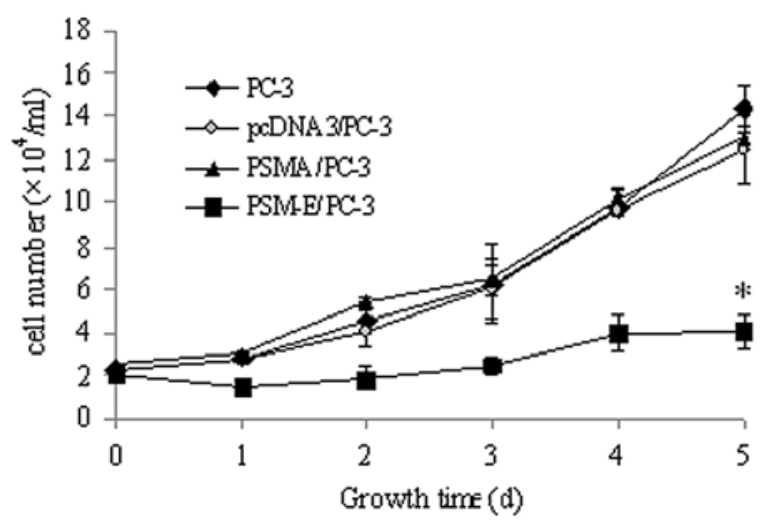

$\mathrm{b}$

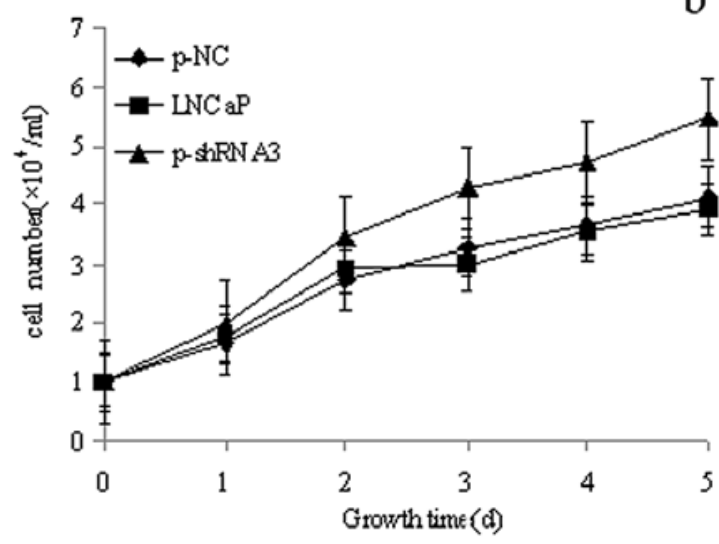

Figure 4. The cells growth curves of PSMA/PC-3 and PSM-E/PC-3 overexpression cells and PSMA/PSM-E interfering LNCap cells. (a) Growth curves of PC- 3 cells transfected by PSMA/PSM-E pcDNA3.0 expression vectors, ${ }^{*} \mathrm{P}<0.05$ compared with other groups. (b) Growth curves of PSMA/PSM-E interfering LNCap cells transfected by p-shRNA3.

Table II. Migration speed of PSM-E/PSMA overexpressed and interfering prostate cancer cells.

\begin{tabular}{|c|c|c|c|c|c|c|c|}
\hline & $\mathrm{PC}-3$ & pcDNA3.0/PC-3 & PSMA/PC-3 & PSM-E/PC-3 & $\mathrm{LNCaP}$ & $\mathrm{p}-\mathrm{NC}$ & p-shRNA3 \\
\hline Cell migration speed $(\mu \mathrm{m} / \mathrm{h})$ & $40.0 \pm 2.65$ & $39.0 \pm 2.65$ & $21.0 \pm 3.61$ & $21.3 \pm 2.08$ & $34.4 \pm 3.69$ & $32.2 \pm 2.99$ & $40.6 \pm 2.44$ \\
\hline P-value & 0.66 & & $0.002^{\mathrm{a}}$ & $0.001^{\mathrm{a}}$ & 0.46 & & $0.019^{\mathrm{b}}$ \\
\hline
\end{tabular}

The P-values of PC-3, PSMA/PC-3 and PSM-E/PC-3 groups were the P-value compared with the pcDNA3.0/PC-3 group (vector negative control), and $\mathrm{P}$-values of $\mathrm{LNCaP}$ and $\mathrm{p}$-shRNA3 group were the $\mathrm{P}$-value compared with the $\mathrm{p}$-NC group (vector negative control). ${ }^{\mathrm{a}} \mathrm{P}<0.01$ compared with vector negative control; ${ }^{\mathrm{b}} \mathrm{P}<0.05$ compared with vector negative control.

control and non-transfected PC-3 negative control groups $(\mathrm{P}<0.01$, Table II, Fig. 5). However, there was no significant difference of the effect of migration suppression between PSM-E/PC-3 and PSMA/PC-3 ( $>0.05)$. The migration speeds of p-shRNA3, p-NC and LNCaP cells were $40.6 \pm 2.44$, $32.2 \pm 2.99$, and $34.4 \pm 3.69 \mu \mathrm{m} / \mathrm{h}$, respectively. The migration speed of p-shRNA3 transfected LNCaP cells was higher than that of $\mathrm{p}-\mathrm{NC}$ and $\mathrm{LNCaP}$ control groups $(\mathrm{P}<0.05$, Fig. 5), which was consistent with the results of PSM-E/PC-3 and PSMA/PC-3 cross-river tests, showing that both PSMA and its new alternatively spliced variant PSM-E had the activity of migration suppression of $\mathrm{PCa}$ cells.

Invasiveness analysis of PSM-E/PSMA overexpressed PC-3 cells and interfering $L N C a P$ cells. The results of transwell assay of PSM-E/PSMA overexpressed PC-3 cells showed that for PSMA/PC-3 group, the cells were the least invasive, and there was significant difference between PSMA/PC-3 group and vector negative control $(\mathrm{P}<0.01)$, and also between PSM-E/ PC-3 group and vector negative control $(\mathrm{P}<0.05$, Table III, Fig. 6a-d). There was also significant difference of the invasive cell number between the PSM-E/PC-3 group and PSMA/ PC-3 ( $\mathrm{P}<0.05$, Table III, Fig. 6), indicating that the effect of invasive suppression of PSMA was stronger than PSM-E. The invasiveness index of PSMA/PC-3 group was $45.10 \%$, and the invasiveness index of PSM-E/PC-3 group was $80.83 \%$. Accordingly, the Transwell assay results of PSM-E/PSMA interfering $\mathrm{LNCaP}$ cells showed that the invasive $\mathrm{LNCaP}$ cell number of $p$-shRNA3 group was significantly higher than the $\mathrm{p}-\mathrm{NC}$ vector negative control group $(\mathrm{P}<0.01)$, and there was no 

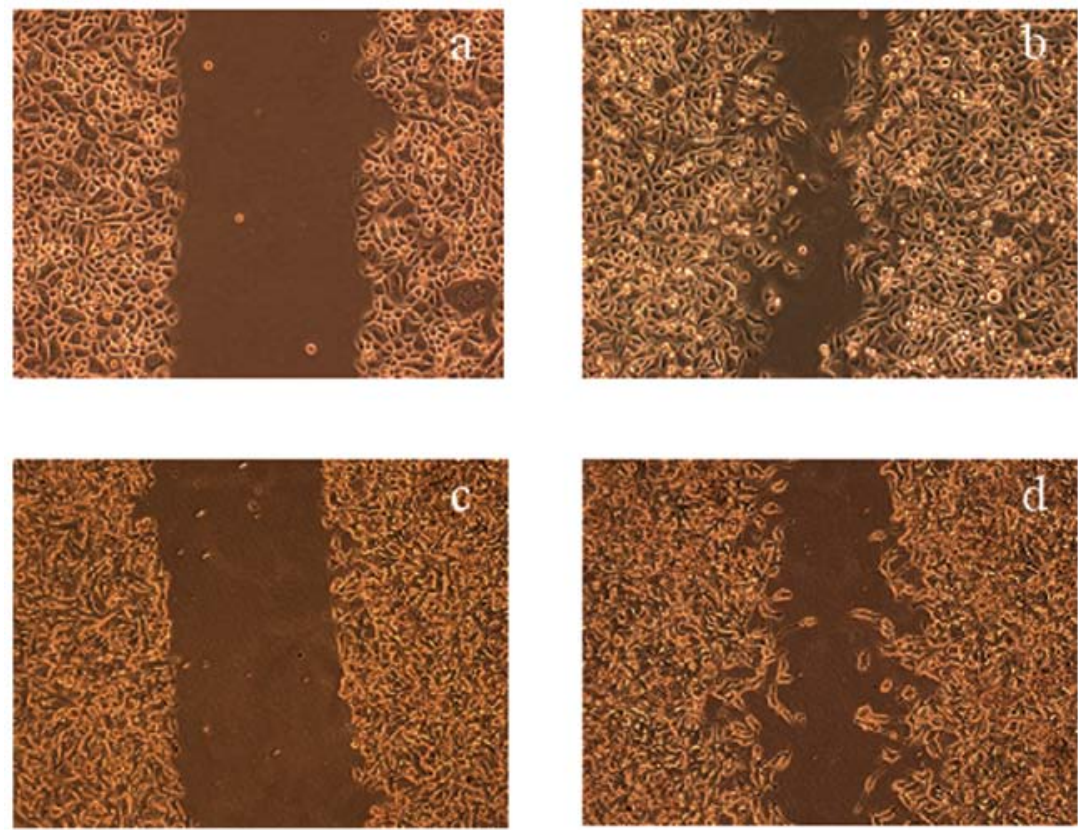

Figure 5. The cross-river analysis of PSM-E/PC-3 cells and p-shRNA3 interfering LNCaP cells. PSMA/PC-3 and PSM-E/PC-3 showed similar migration speed, and both of them showed slower migration speed than vector negative control and PC-3 negative control. Accordingly, p-shRNA3 interfering LNCaP cells migrated faster than p-NC transfected LNCaP cells. (a) PSM-E/PC-3 cells at $24 \mathrm{~h}$ (phase contrast, $\mathrm{x} 100$ ). (b) pcDNA3.0/PC-3 cells at $24 \mathrm{~h}$ (phase contrast, $\mathrm{x} 100$ ). (c) p-NC transfected LNCaP cells at $24 \mathrm{~h}$ (phase contrast, $\mathrm{x} 100$ ). (d) p-shRNA3 interfering LNCaP cells at $24 \mathrm{~h}$ (phase contrast, $\mathrm{x} 100$ ).

Table III. Invasive cell number of PSM-E/PSMA overexpressed and interfering prostate cancer cells.

\begin{tabular}{lcc}
\hline Groups & $\begin{array}{c}\text { Invasive cell number } \\
\text { (cells/HP) }\end{array}$ & P-value \\
\hline PC-3 & $19.47 \pm 1.90$ & 0.466 \\
pcDNA3.0/PC-3 & $18.47 \pm 1.00$ & \\
PSMA/PC-3 & $8.33 \pm 2.51$ & $0.003^{\mathrm{a}}$ \\
PSM-E/PC-3 & $14.93 \pm 1.93$ & $0.048^{\mathrm{a}}$ \\
LNCaP & $14.88 \pm 3.38$ & 0.358 \\
p-NC & $11.62 \pm 2.38$ & \\
p-shRNA3 & $25.60 \pm 1.91$ & $0.002^{\mathrm{b}}$ \\
\hline
\end{tabular}

The P-values of PC-3, PSMA/PC-3 and PSM-E/PC-3 groups were $\mathrm{P}$-value compared with the pcDNA3.0/PC-3 group (vector negative control), and P-values of LNCaP and p-shRNA3 group were the $\mathrm{P}$-value compared with the $\mathrm{p}-\mathrm{NC}$ group (vector negative control). ${ }^{\text {aa }}<0.05$ compared with vector negative control; ${ }^{b} \mathrm{P}<0.05$ compared with vector negative control.

significant difference between the $\mathrm{p}-\mathrm{NC}$ group and the nontransfected LNCaP group ( $\mathrm{P}>0.05$, Table III, Fig. 6e-f). The invasiveness index of p-shRNA3 was $220.3 \%$. The invasiveness analysis showed that PSM-E had the effect of invasive suppression, but the activity was weaker than PSMA.

\section{Discussion}

Alternative splicing is a fundamental mechanism in human differential gene expression, and can also be seen in cancer cells $(16,17)$. Recent genome wide analysis of alternative splicing indicates that as much as $70 \%$ of human genes may have alternative spliced forms $(17,20,21)$. Changes in splice site selection and the production of splice variants have been observed in various types of cancer and may affect tumor progression and susceptibility to cancer. Four splice variants of PSMA have been reported as PSM', PSM-C, PSM-D, and PSM-E (18-19,22-23). PSM' is located in the cytoplasm and produces a protein that lacks the intracellular and transmembrane domains of PSMA $(18,22,23)$. PSM-C generates a protein identical to PSM'. PSM-D has a new translation initiation start site in exon 1c, followed by 42 novel amino acids and the rest of the PSMA protein in frame (22). There was evidence that the differential expression of PSMA and the above three alternatively spliced variants may be related to the progression of PCa (24). PSM-E is a newly found splice variant, with a $97 \mathrm{bp}$ of exon 1c insertion (similar to that of PSM-D) and a 93 bp deletion that was not seen in PSMA or in any of the known PSMA splice variants (19). Our preliminary study showed its high tissue specificity and pathological specificity, but its role in PCa progression is still uncertain. Since the proliferation, invasiveness and migration ability of the malignant tumor cells in vitro could reflect, to a large extent, the process of tumor growth and progression in vivo, in this study, we constructed PSM-E and PSMA expressing cell clones, using PC-3 as the target cell line because PC-3 is a known PCa cell line that does not express PSMA or its splice variants, to explore the possible effect of PSM-E on the proliferation, invasiveness and migration of $\mathrm{PCa}$ cells. Accordingly, the PSMA and splice variants positive PCa cell line LNCaP was used to construct the shRNA interference cell model, to further verify the results of PSM-E/PSMA overexpressed PC-3 cells. Since some parts of the PSMA gene were homologous with other genes like transferrin receptor and M28 

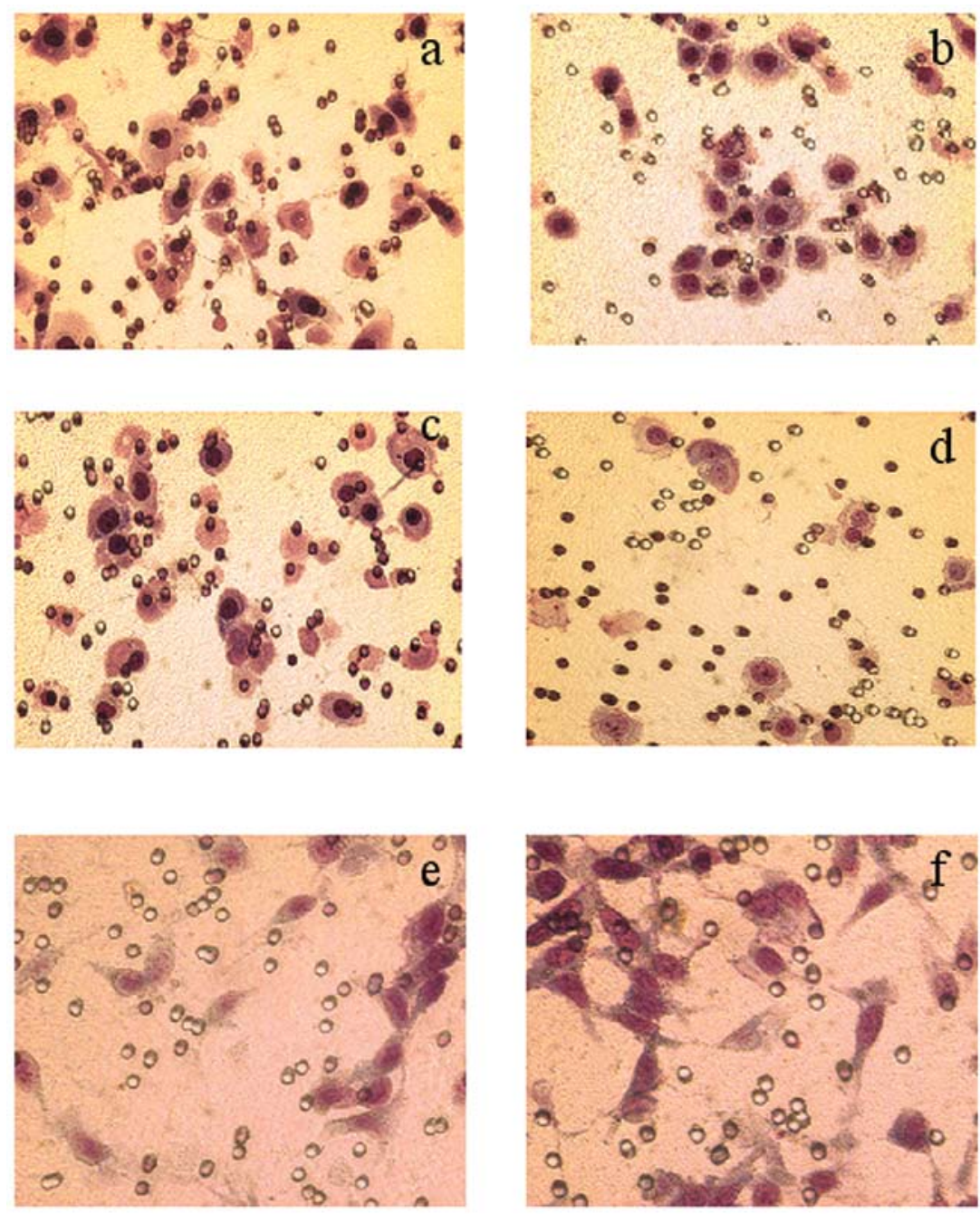

Figure 6. The transwell invasiveness analysis of PSM-E/PSMA overexpressed PC-3 cells and PSMA and its spliced variants interfering LNCaP cells. Cells crossing the membrane were stained by Wright-Giemsa staining solution. (a) Invasive PC-3 negative control cells (Wright-Giemsa staining, x200).(b) Invasive pcDNA3.0/PC-3 vector negative control cells (Wright-Giemsa staining, x200). (c) Invasive PSM-E/PC-3 cells (Wright-Giemsa staining, x200). (d) Invasive PSMA/PC-3 cells (Wright-Giemsa staining, x200). (e) Invasive LNCaP cells transfected by p-NC (Wright-Giemsa staining, x400). (f), Invasive LNCaP cells transfected by $\mathrm{p}$-shRNA3 (Wright-Giemsa staining, $\mathrm{x} 400$ ).

family of co-catalytic aminopeptidases $(3,8,25)$, specificity and effectiveness were two capital considerations in siRNA design. Because PSMA, PSM-E and other splice variants are extremely homologous, it is hard to design siRNA that could distinguish PSM-E from PSMA and other splice variants and at the same time. Therefore, the siRNA used in this study was specific to PSMA and its splice variants.

The identification experiments showed that the PSMA and PSM-E gene had been successfully transfected into PC-3 cells, and can be successfully expressed at both the mRNA and protein level, as confirmed by RT-PCR and Western blot analysis. Three shRNA fragments were obtained based on the siRNA design principles of Elbashir et al (26), recognization sites as 869 bp, 1589 bp, and 2149 bp, respectively. Their interference effect was evaluated using RT-PCR and Western blotting in LNCaP cells. After expression, all 3 shRNA showed inhibition effect at both mRNA and protein level. The mRNA inhibition rates of the constructed three 2.1-U6-neo-shRNA expression vectors (namely p-shRNA1,2,3) were $33.15 \%$, $9.26 \%$ and $41.97 \%$, respectively, and the protein inhibition rate was $26.26 \%, 6.47 \%$ and $40.69 \%$, respectively, similar to other reported interfering RNAs (27-28). So 2.1-U6-neo-shRNA3 (namely p-shRNA3) was chosen in the following analysis, because it showed the maximum interference effect in expression of PSMA and its spliced variants.

To further evaluate the function of PSM-E/PSMA in the biological behavior of the PCa cells, proliferation analysis (tumor doubling time), invasiveness tests (transwell test) and migration tests (cross-river test) were carried out. In this study, there was no statistical difference of tumor doubling time between PSMA/PC-3 group and the vector negative control group, indicating that PSMA did not affect the cell growth significantly $(\mathrm{P}>0.05)$, whereas in PSM-E/PC-3 cells, cell proliferation was significantly suppressed $(\mathrm{P}<0.05$, Table I), showing that PSM-E had the ability to suppress PCa cell proliferation. Although compared with non-transfected LNCaP cells and p-NC group, no significant difference of proliferation was observed in p-shRNA3 group, it also showed a trend of cell doubling time shortening. We presume that in PSMA and its spliced variants interfering LNCaP cells, the prolif- 
eration suppression effect may be covered up by PSMA and other alternative spliced variants. The mechanism of PSM-E suppressing cell proliferation will be further explored.

Invasiveness and metastasis are two major abilities of malignant tumors. Since PSM-E has somewhat different amino acid composition, what is the effect of PSM-E on cell migration and invasiveness of PCa cells? In our study, the cross-river tests showed that the migration speed of PSM-E/PC-3 group was similar to that of PSMA/PC-3 group ( $\mathrm{P}>0.05)$, and both were significantly slower than the vector negative control group and PC-3 negative control ( $\mathrm{P}<0.01$, Table II, Fig. 5), indicating that PSM-E, similar to PSMA, could suppress the migration of PCa cells. Accordingly, the migration speed of p-shRNA3 transfected LNCaP cells was higher than that of p-NC and LNCaP control groups ( $\mathrm{P}<0.05$, Fig. 5), consistent with the results of PSM-E/PC-3 and PSMA/PC-3 cross-river tests, further confirming and showing that both PSMA and its alternatively spliced variant PSM-E had the activity of migration suppression of PCa cells.

The role of PSMA in tumor migration and invasiveness is still uncertain, and contradictory results exist. A recent study of Ghosh et al revealed that PSMA could suppress the PC-3 cell invasiveness (29). In our study, similar phenomena were observed in PSMA/PC-3 transwell test $(\mathrm{P}<0.05$ compared with the vector negative control), and PSM-E/PC-3 also showed the effect of invasive suppression $(\mathrm{P}<0.05$, Table III, Fig. 6a-d), but the effect was weaker than PSMA $(\mathrm{P}<0.05)$. This was not an exceptional phenomenon in splice variants. It can be observed in other tumor-associated genes. The KAI1 gene is a PCa-suppressing gene that could suppress tumor metastasis, and the encoded protein of one of its splice variant (which lacked exon 7) showed weaker ability in suppressing tumor metastasis (30). To our knowledge, this is the first report that such change can also be observed in PSMA and its splice variant, PSM-E. When PSMA and its splice variants were blocked, the invasiveness index of $\mathrm{LNCaP}$ increased dramatically $(\mathrm{P}<0.01)$, which further confirmed the invasiveness inhibition activity observed in PSM-E/PC-3 and PSMA/PC-3 cells.

It is of interest that the proliferation suppression effect of PSM-E was stronger than PSMA, whereas its invasiveness suppression effect was weaker than PSMA. Since PSM-E differ only a $97 \mathrm{bp}$ insertion and a 93 bp deletion from PSMA, the mechanism behind cell biological behavior difference may lie in their structure difference. There was evidence that the activity of PSMA in suppressing tumor migration and invasiveness is due to its enzymatic activity, the NAALADase (29). It was revealed that if the PSMA mutant without NAALADase activity was transfected into PC-3 cells, the cell invasiveness would be higher than that of the PC-3 cells transfected by PSMA with NAALADase activity, and if enzymatic inhibitor was applied in PSMA positive cells, the cell invasiveness would be enhanced (29). The NAALADase activity can be affected by many factors such as the $\mathrm{N}$ terminal glycosylation and protein homodimer structure (31). The exon 18 deletion of PSM-E would cause a shorter extracellular part of PSM-E protein, and there was evidence that lack of exon 18 may affect homodimer formation and cause the loss of NAALADase activity (32), thus, we believed that the weaker ability of PSM-E in suppressing PC-3 invasiveness and stronger effect on proliferation suppression may be due to this. Further study should be needed for direct evidence of its enzymatic activity, in order to know more about its function and possible use in the regulation of tumor progression and $\mathrm{PCa}$ treatment.

PSM-E is a newly found alternative splice variant of PSMA. In this study, it was revealed that PSM-E could affect the biological behavior of PCa cells. It is able to suppress PCa cell proliferation, migration and invasiveness. It had stronger effect of proliferation suppression than PSMA, similar effect of migration suppression but weaker effect of invasiveness suppression. The effect may be related to its TfR and NAALADase activity. To our knowledge, this is the first report on the effect of PSM-E on PCa cell proliferation, migration and invasiveness. Our findings may provide new insights into the relationships between the spliced variant construction and activity of PSMA and PSM-E, and may have important significance for the mechanism study of PSM-E contribution to cancer progression.

\section{Acknowledgements}

This study was supported by grants of National Natural Science Foundation, China (no. 81071760, 30772503, 30371426), and Guangdong Natural Science Foundation, China (no. 021907).

\section{References}

1. Jemal A, Siegel R, Ward E, Murry T, Xu J and Thun MJ: Cancer statistics. CA Cancer J Clin 57: 43-66, 2007.

2. Nelson WG, De Marzo AM and Isaacs WB: Prostate cancer. N Engl J Med 349: 366-381, 2003.

3. Israeli RS, Powell CT, Fair WR and Heston WD: Molecular cloning of a complementary DNA encoding a prostate-specific membrane. Cancer Res 53: 227-230, 1993.

4. Denmeade SR, Sokoll LJ, Dalrymple S, Rosen DM, Gady AM, Bruzek D, Ricklis RM and Isaacs JT: Dissociation between androgen responsiveness for malignant growth vs. expression of prostate specific differentiation markers PSA, hK2, and PSMA in human prostate cancer models. Prostate 54: 249-257, 2003.

5. Karan D, Kelly DL, Rizzino A, Lin MF and Batra SK: Expression profile of differentially regulated genes during progression of androgen-independent growth in human prostate cancer cells. Carcinogenesis 23: 967-975, 2002.

6. Lapidus, RG, Tiffany, CW, Isaacs, JT and Slusher BS: Prostatespecific membrane antigen (PSMA) enzyme activity is elevated in prostate cancer cells. Prostate 45: 350-354, 2000.

7. Ross JS, Sheehan CE, Fisher HA, Kaufman RP Jr, Kaur P, Gray K, Webb I, Gray GS, Mosher R and Kallakury BV: Correlation of primary tumor prostate-specific membrane antigen expression with disease recurrence in prostate cancer. Clin Cancer Res 9: 6357-6362,2003.

8. Ghosh A and Heston WD: Tumor target prostate specific membrane antigen (PSMA) and its regulation in prostate cancer. J Cell Biochem 91: 528-539, 2004.

9. Kinoshita Y, Kuratsukuri K, Landas S, Imaida K, Rovito PM Jr, Wang CY and Haas GP: Expression of prostate-specific membrane antigen in normal and malignant human tissues. World J Surg 30: 628-636, 2006.

10. O'Keefe DS, Bacich DJ and Heston WD: Comparative analysis of prostate-specific membrane antigen (PSMA) versus a prostate-specific membrane antigen-like gene. Prostate 58: 200-210, 2004.

11. Chang SS, Reuter VE, Heston WD, Bander NH, Grauer LS and Gaudin PB: Five different anti-prostate-specific membrane antigen (PSMA) antibodies confirm PSMA expression in tumor- associated neovasculature. Cancer Res 59: 3192-3198, 1999.

12. Chang SS, Reuter VE, Heston WD and Gaudin PB: Metastatic renal cell carcinoma neovasculature expresses prostate-specific membrane antigen. Urology 57: 801-805, 2001. 
13. Liu T, Jabbes M, Nedrow-Byers JR, Wu LY, Bryan JN and Berkman CE: Detection of prostate-specific membrane antigen on HUVECs in response to breast tumor-conditioned medium. Int J Oncol 38: 1349-1355, 2011.

14. Ma Q, Safar M, Holmes E, Wang Y, Boynton AL and Junghans RP: Anti-prostate specific membrane antigen designer T cells for prostate cancer therapy. Prostate 61: 12-25, 2004.

15. Dhar S, Kolishetti N, Lippard SJ and Farokhzad OC: Targeted delivery of a cisplatin prodrug for safer and more effective prostate cancer therapy in vivo. Proc Natl Acad Sci USA 108: 1850-1855, 2011.

16. Keren H, Lev-Maor G and Ast G: Alternative splicing and evolution: diversification, exon definition and function. Nat Rev Genet 11: 345-355, 2010.

17. Kalnina Z, Zayakin P, Silina $\mathrm{K}$ and Line A: Alterations of pre-mRNA splicing in cancer. Genes Chromosomes Cancer 42: 342-357, 2005.

18. Su SL, Huang IP, Fair WR, Powell CT and Heston WD: Alternatively spliced variants of prostate-specific membrane antigen RNA: ratio of expression as a potential measurement of progression. Cancer Res 55: 1441-1443, 1995.

19. Cao KY, Mao XP, Wang DH, Xu L, Yuan GQ, Dai SQ, Zheng BJ and Qiu SP: High expression of PSM-E correlated with tumor grade in prostate cancer: a new alternatively spliced variant of prostate-specific membrane antigen. Prostate 67: 1791-1800, 2007.

20. Johnson JM, Castle J, Garrett-Engele P, Kan Z, Loerch PM, Armour CD, Santos R, Schadt EE, Stoughton R and Shoemaker DD: Genome-wide survey of human alternative pre-mRNA splicing with exon junction microarrays. Science 302 : 2141-2144, 2003

21. Casado-Vela J, Cebrian A, del Pulgar MT, Sanchez-Lopez E, Vilaseca M, Menchen L, Diema C, Selles-Marchart S, Martinez-Esteso MJ, Yubero N, Bru-Martinez R and Lacal JC: Lights and shadows of proteomic technologies for the study of protein species including isoforms, splicing variants and protein post-translational modifications. Proteomics 11: 590-603, 2011

22. O'Keefe DS, Bacich DJ and Heston WD: Prostate specific membrane antigen. In: Prostate Cancer, Biology, Genetics, and the New Therapeutics. Chung LWK, Issacs WB, Simons JW (edit). Humana Press, Totowa, NJ, pp307-326, 2001.
23. Mlcochova P, Barinka C, Tykvart J Sacha P and Konvalinka J: Prostate-specific membrane antigen and its truncated form PSM'. Prostate 69: 471-479, 2009.

24. Schmittgen TD, Teske S, Vessella RL, True LD and Zakrajsek BA: Expression of prostate specific membrane antigen and three alternatively spliced variants of PSMA in prostate cancer patients. Int J Cancer 107: 323-329, 2003.

25. Rawlings ND and Barrett AJ: Structure of membrane glutamate carboxypeptidase. Biochem Biophys Acta 1339: 247-252, 1997.

26. Elbashir SM, Harborth J, Weber K and Tuschl T: Analysis of gene function in somatic mammalian cells using small interfering RNAs. Methods 26: 199-213, 2002.

27. Gao L, Zhang L, Hu J, Li F, Shao Y, Zhao D, Kalvakolanu DV, Kopecko DJ, Zhao X and Xu DQ: Down-regulation of signal transducer and activator of transcription 3 expression using vector-based small interfering RNAs suppresses growth of human prostate tumor in vivo. Clin Cancer Res 11: 6333-6341, 2005.

28. Shen HL, Xu W, Wu ZY, Zhou LL, Qin RJ and Tang HR: Vectorbased RNAi approach to isoform-specific downregulation of vascular endothelial growth factor (VEGF)165 expression in human leukemia cells. Leuk Res 31: 515-521, 2007.

29. Ghosh A, Wang XN, Klein E and Heston WD: Novel role of prostate-specific membrane antigen in suppressing prostate cancer invasiveness. Cancer Res 65: 727-731, 2005.

30. Lee JH, Seo YW, Park SR, Kim YJ and Kim KK: Expression of a splice variant of KAI1, a tumor metastasis suppressor gene, influences tumor invasion and progression. Cancer Res 63: 7247-7255, 2003.

31. Schiilke N, Varlamova OA, Donovan GP, Ma D, Gardner JP, Morrissey DM, Arrigale RR, Zhan C, Chodera AJ, Surowitz KG, Maddon PJ, Heston WD and Olson WC: The homodimer of prostate-specific membrane antigen is a function target for cancer therapy. Proc Natl Acad Sci USA 22: 12590-12591, 2003.

32. Williams T and Kole R: Analysis of prostate-specific membrane antigen splice variants in LNCap cells. Oligonucleotides 16: 186-195, 2006 